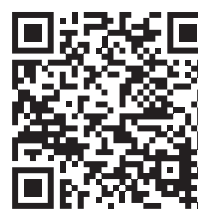

\title{
Trastorno del sueño. Un problema frecuente en los pacientes pediátricos diagnosticados con asma
}

\author{
Norma Mariana Tena-Fernel, ${ }^{*}$ Luis Sandoval-Jurado, ${ }^{\ddagger}$ Zoila Inés Ceballos-Martínez, ${ }^{\S}$ \\ María Valeria Jiménez-Báez," Juan Carlos Espinoza-Rodríguez*
}

\section{RESUMEN}

Objetivo: Estimar la frecuencia de trastornos del sueño en la población infantil de un Hospital de Segundo Nivel en Quintana Roo. Material y métodos: Estudio transversal comparativo en niños con diagnóstico de asma confirmado por el médico pediatra de la consulta externa. Como instrumento de medición se utilizó la escala de Trastornos del Sueño para Niños de Bruni con un punto de corte para presencia de trastorno de $\geq$ 39. Resultados: Se incluyeron 146 pacientes de la Consulta Externa de Pediatría, 73 niños con diagnóstico de asma y 73 niños sin asma. Los trastornos del sueño estuvieron presentes en $71.2 \%$ de los niños con asma, observando un riesgo en los niños asmáticos cinco veces mayor de presentar trastornos del sueño $(\mathrm{RP}=5.05 \mathrm{IC} 95 \%$ : 2.50-10.21). El alérgeno con mayor frecuencia entre los pacientes con asma y trastorno del sueño fue el contar con mascota (con pelo) con 68.1\% $(p<0.05)$. Conclusión: La presencia de trastorno del sueño en la población infantil con asma es mayor a lo referido por otros autores; sin embargo, en la práctica clínica diaria no es considerado como un elemento para tratamiento, condicionando un retraso en el control de la enfermedad que influye en el comportamiento y funcionamiento cognitivo.

Palabras clave: Trastorno del sueño, asma, población pediátrica, alérgenos.

\section{ABSTRACT}

Objective: To estimate the frequency of sleep disorders in the child population of a second level hospital in Quintana Roo. Material and methods: Comparative cross-sectional study in children diagnosed with asthma confirmed by the hospital pediatrician. The Bruni Child Sleep Disorder Scale was used as a measuring instrument with a cut-off point for the presence of a disorder of $\geq 39$. Results: Were included 146 patients from the pediatric medical consulting room, 73 children diagnosed with asthma and 73 children without asthma. Sleep disorders were present in $71.2 \%$ of children with asthma, with a 5-fold higher risk of asthmatic children than sleep disorders ( $P R=5.05,95 \% \mathrm{Cl}$ : 2.50-10.21). The most frequent allergen among patients with asthma and sleep disorder was having a pet (with hair) with $68.1 \%(p<0.05)$. Conclusion: The presence of sleep disorder in children with asthma is higher than that reported by other authors, however in daily clinical practice, it is not considered as an element for treatment, conditioning a delay in the control of the disease and a possible influence in behavior and cognitive functioning.

Keywords: Sleep disorder, asthma, pediatric population, allergens.

\footnotetext{
* Unidad de Medicina Familiar Núm. 16.

* Coordinación Auxiliar de Investigación en Salud.

$\S$ Departamento de Pediatría, Hospital General Regional Núm. 17.

॥ Coordinación de Planeación y Enlace Institucional.
} 
Vol. 29, Núm. 2 • Mayo-Agosto 2020

\section{INTRODUCCIÓN}

El asma, definida por La Organización Mundial de la Salud es una enfermedad crónica que se caracteriza por ataques recurrentes de disnea y sibilancias, los cuales varían en severidad y frecuencia. ${ }^{1}$

En México el asma se ha ubicado como una de las primeras 20 causas de morbilidad, la población más vulnerable en $28.5 \%$ resulta ser el grupo que oscila entre los cinco y los 14 años de edad. ${ }^{2}$ En Quintana Roo en niños de edad preescolar a preparatoria se encontró una prevalencia de $14 \%{ }^{3}$

Las enfermedades respiratorias como el asma, la hipertrofia de adenoides, amígdalas o cornetes y la atopia, solas o combinadas son factores de riesgo para el desarrollo del síndrome de apnea obstructiva del sueño (SAOS). La fisiopatología de estos desórdenes coincide debido a que ambas son afectadas por un proceso inflamatorio, así como neuronas aferentes y factores anatómicos como la obesidad, estos síntomas nocturnos pueden causar la interrupción del sueño y una pobre calidad del mismo con repercusión en el crecimiento. ${ }^{4}$ Además de representar un factor de riesgo para la presencia de trastornos del sueño. ${ }^{5}$

Estos trastornos son definidos con base en la Guía de Práctica Clínica como una serie de alteraciones relacionadas con el proceso de dormir, pueden presentarse tanto en las etapas de inicio-mantenimiento como durante el ciclo sueño-vigilia. ${ }^{6}$ En el sueño infantil la proporción de sueño REM y NREM se distribuye en partes iguales, cercano al $50 \%$ por cada uno, a diferencia del adulto joven en el cual, el sueño REM ocupa $25 \%$ del total de la noche. ${ }^{7}$ Existen múltiples trastornos del sueño, los principales son el síndrome apneas-hipoapneas del sueño (SAHS) y el síndrome de apnea obstructiva del sueño (SAOS). ${ }^{8}$

En pacientes asmáticos de cuatro a 18 años de edad se encontró asociación entre los trastornos del sueño, asma moderada-severa y obesidad. ${ }^{4,9}$ En población escolar comprendida entre los cinco y 12 años de edad se observó una asociación entre los trastornos del sueño y factores predisponentes de asma; se demostró que sí existe una relación positiva entre estas dos variables con un $\mathrm{OR}=1.58 .^{10}$

La presencia de trastornos del sueño en niños y adolescentes con obesidad, entre cinco y 14 años y un grupo control, evaluada por la escala de trastornos de sueño de Bruni, se observó que los sujetos obesos tienen mayor riesgo de desarrollar trastornos del sueño con un $\mathrm{OR}=1.9 ; 95 \%$ IC $1.8-3.2^{11}$ y la prevalencia de los trastornos de sueño fue de $62.3 \% .^{12}$

El estudio PIAMA (Prevention and Incidence of Asthma and Mite Allergy) realizado en niños, encontró que aquellos pacientes con síntomas frecuentes de asma presentaron mayor cansancio y somnolencia diurna comparado con pacientes asintomáticos y con sintomatología leve; sin embargo, no presentaron diferencias al evaluar los trastornos del sueño. ${ }^{13}$

El objetivo del estudio es estimar la frecuencia de trastornos del sueño en la población infantil de un Hospital de Segundo Nivel en Quintana Roo.

\section{MATERIAL Y MÉTODOS}

Se realizó un estudio comparativo en una población infantil de la consulta externa de pediatría de un Hospital de Segundo Nivel de Atención, con una edad comprendida entre los dos y los 15 años de edad. Se conformaron dos grupos, el primero con diagnóstico de asma emitido por el médico pediatra y el segundo con las mismas características, pero sin el diagnóstico de asma o alguna enfermedad de la vía respiratoria.

Previo consentimiento informado firmado por alguno de los padres o quien acudió con el niño a su cita en pediatría, se les comentó el objetivo del estudio y en

Tabla 1: Características de la población de estudio en relación al diagnóstico de asma y presencia de trastorno del sueño.

\begin{tabular}{|c|c|c|c|c|c|c|}
\hline \multirow[b]{2}{*}{ Característica } & \multicolumn{3}{|c|}{ Diagnóstico de asma n (\%) } & \multicolumn{3}{|c|}{ Trastornos del sueño n (\%) } \\
\hline & Sí $(n=73)$ & Vo $(n=73)$ & Total & Sí $(n=76)$ & No $(n=70)$ & Total \\
\hline \multicolumn{7}{|l|}{ Grupo etario } \\
\hline Preescolar & $28(38.4)$ & $43(58.9)$ & $71(48.6)$ & $32(42.1)$ & $39(55.7)$ & $71(48.6)$ \\
\hline Escolar & $39(53.4)$ & $22(30.1)$ & $61(41.8)$ & $35(46.1)$ & $26(37.1)$ & $61(41.8)$ \\
\hline Adolescente & $6(8.2)$ & $8(11.0)$ & $14(9.6)$ & $9(11.8)$ & $5(7.1)$ & $14(9.6)$ \\
\hline \multicolumn{7}{|l|}{ Sexo } \\
\hline Hombre & $43(58.9)$ & $39(53.4)$ & $82(56.2)$ & $46(60.5)$ & $36(51.4)$ & $82(56.2)$ \\
\hline Mujer & $30(41.1)$ & $34(46.6)$ & $64(43.8)$ & $30(39.5)$ & $34(48.6)$ & $64(43.8)$ \\
\hline \multicolumn{7}{|l|}{ IMC } \\
\hline Sobrepeso y obesidad & $22(30.1)$ & $16(21.9)$ & $38(26.0)$ & $22(28.9)$ & $16(22.9)$ & $38(26.0)$ \\
\hline Normopeso & $51(69.9)$ & $57(78.1)$ & $108(74.0)$ & $54(71.1)$ & 54 (77.1) & $108(74.0)$ \\
\hline
\end{tabular}


Vol. 29, Núm. 2・ Mayo-Agosto 2020

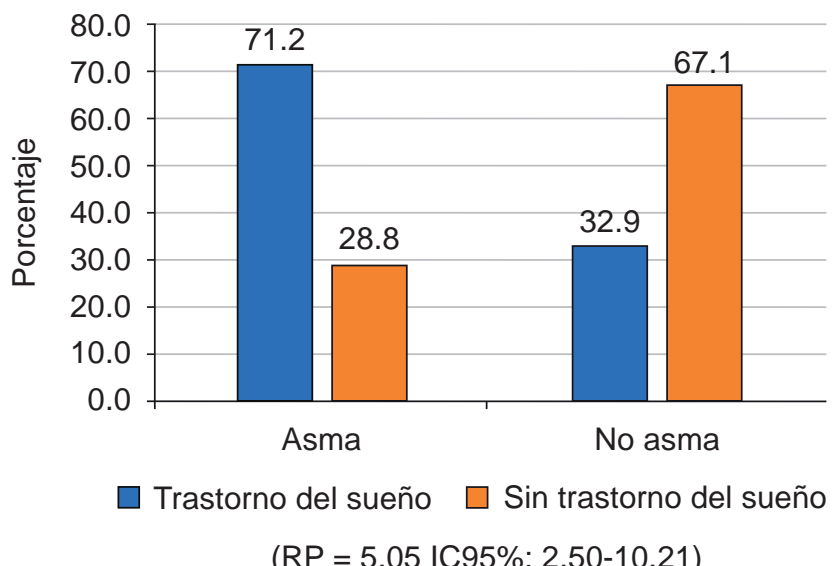

Figura 1: Trastorno del sueño en relación al diagnóstico de asma.

qué consistiría su participación. Asimismo, se comentó que se respetaría en todo momento el anonimato de los participantes y el manejo confidencial de los datos y resultados generados en la investigación. Posteriormente, se les invitó a un área física adjunta al consultorio y se les otorgaron dos cuestionarios, uno de ellos con datos personales, sociodemográficos y de la enfermedad (asma), mientras que el segundo era la escala de Trastornos del Sueño para Niños de Bruni (Sleep Disturbance Scale for Children, SDSC) que consta de 26 reactivos que evalúan: trastornos para iniciar y mantener el sueño, trastornos de respiración durante el sueño, trastornos de activación y pesadillas, trastornos en la transición sueño-despertar y trastornos por excesiva somnolencia e hiperhidrosis. La escala fue diseñada para realizar la pesquisa de trastornos del sueño en los últimos seis meses. A cada uno de los 26 ítems se les dio un puntaje del 1 al 5; al obtener un puntaje igual o mayor a 39 puntos se sospecha la presencia de trastornos del sueño. ${ }^{14}$

La respuesta para ambos instrumentos fue otorgada por los padres o quienes acudieron con el menor a la consulta, si existía alguna duda el investigador la aclaró en el momento. Los padres o tutores de los pacientes que estuvieron de acuerdo en participar en el estudio se les pidió como criterio de inclusión el saber leer y escribir. Aquéllos que no eran derechohabientes no fueron incluidos en el estudio. Los cuestionarios no contestados en su totalidad fueron eliminados. Se tomaron en cuenta la edad, género, peso y talla del paciente, así como el trastorno de sueño, asma, obesidad, familiares directos con asma, lugar donde duerme, número de personas con las que duerme en el mismo espacio, alérgenos, uso de biberón y/o chupón, rinitis alérgica, amigdalectomía, enuresis y tratamiento farmacológico utilizado.
Se estimó un tamaño de muestra para comparación de proporciones con un nivel de confianza del 95\%, un poder estadístico del 90\%, una proporción esperada en el grupo de estudio del 33.0 y $12.0 \%$ para el otro grupo. Con $10 \%$ estimado de pérdidas, se estimó una muestra de 73 niños para cada grupo. Se realizó un muestreo por cuota hasta completar el número requerido para cada grupo. Se estimaron los riesgos mediante la razón de momios con un intervalo de confianza del 95\%. La captura y análisis se realizó en el software estadístico SPSS.

\section{RESULTADOS}

La población estudiada comprendió un total de 146 pacientes, de los cuales $56.2 \%$ correspondieron al género masculino; el promedio de edad fue de 6.8 años (DE \pm 3.41$)$. Al estratificar por grupo etario se observó una mayor frecuencia para el grupo de preescolares con $48.6 \%$ y sólo $9.6 \%$ para adolescentes; de acuerdo al IMC, $26.0 \%$ presentó sobrepeso y obesidad. Del total de la población $52.1 \%$ tuvieron un resultado positivo en la encuesta para trastornos del sueño de Bruni; en el que $46.1 \%$ correspondió a escolares; $60.5 \%$ al género masculino y $71.1 \%$ presentaron normopeso de acuerdo al IMC para edad (Tabla 1). Al estimar la frecuencia de trastorno del sueño en niños con diagnóstico de asma, se observó que estaba presente en 71.2 y $32.9 \%$ en quienes no presentaban esta patología $(p<$ 0.05). Al estimar el riesgo para este grupo se observó que los niños con diagnóstico de asma tienen cinco veces más riesgo de presentar trastornos del sueño que aquéllos sin este padecimiento $(\mathrm{RP}=5.05$ IC95\%: 2.50-10.21) (Figura 1). La presencia de rinitis probable alérgica en los pacientes con asma fue de $24.7 \%$ y al evaluar el trastorno del sueño en relación a este padecimiento se observó que $85.7 \%$ de los niños con

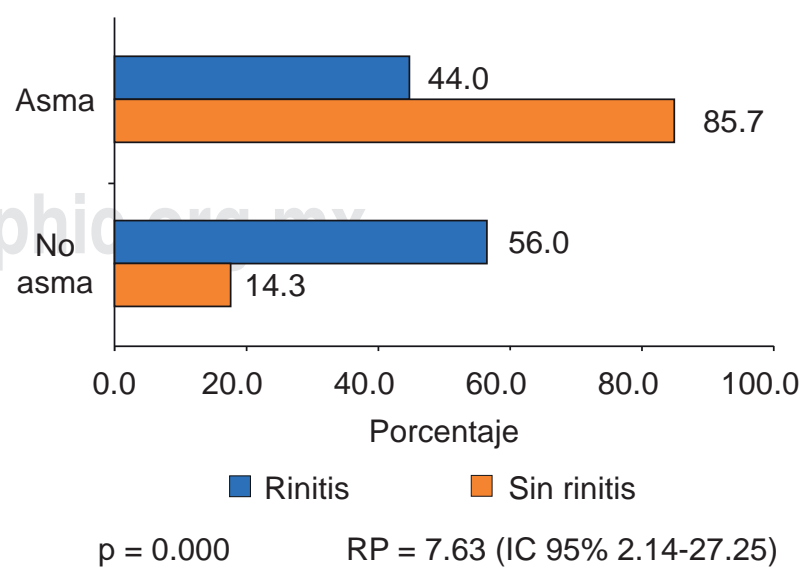

Figura 2: Trastorno del sueño en relación al diagnóstico de rinitis. 
Vol. 29, Núm. 2 • Mayo-Agosto 2020

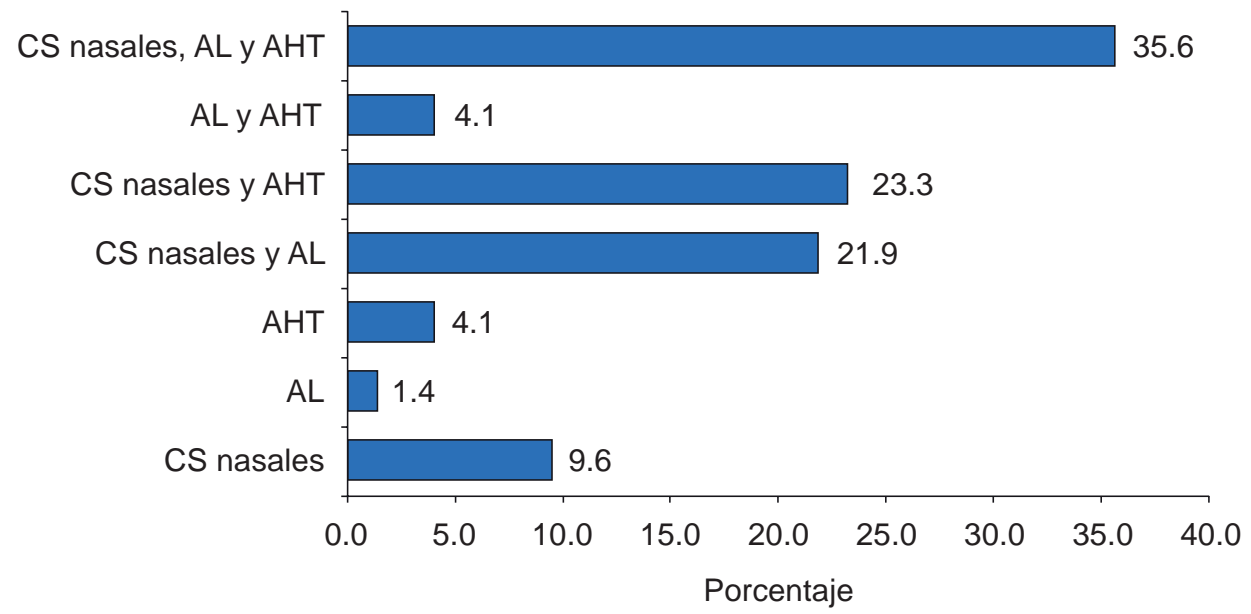

Figura 3:

Medicamentos utilizados en el tratamiento del asma. CS nasales $=$ corticosteroides nasales, $A L=$ antileucotrienos, $A H T=$ antihistamínicos.
Tabla 2: Tiempo de tratamiento y presencia del trastorno del sueño.

\begin{tabular}{lrrrr}
\hline & \multicolumn{4}{c}{ Trastorno del sueño $n(\%)$} \\
\cline { 2 - 4 } \\
\cline { 2 - 4 } $\begin{array}{l}\text { Tiempo de } \\
\text { tratamiento para asma }\end{array}$ & Sí $(\mathrm{n}=52)$ & No $(\mathrm{n}=21)$ & Total & $\mathrm{p}^{*}$ \\
\hline$\leq 1$ año & $12(50.0)$ & $12(50.0)$ & 24 & \\
2 a 5 años & $20(80.0)$ & $5(20.0)$ & 25 & 0.019 \\
$>5$ años & $20(83.3)$ & $4(16.7)$ & 24 & \\
\hline
\end{tabular}

${ }^{*} \chi^{2}$ test.

diagnóstico de rinitis presentaron trastorno del sueño $(\mathrm{p}<0.05)$ (Figura 2).

Dentro del grupo de niños con diagnóstico de asma, el tratamiento más utilizado fue del grupo de los corticosteroides nasales e inhalada en combinación con antileucotrienos y antihistamínicos con $35.6 \%$ y el menor fue el de únicamente antileucotrienos con 1.4\% (Figura 3). Se estimó el tiempo de tratamiento para esta patología y la frecuencia en la presencia de trastorno del sueño; se apreció que en los niños con más de cinco años de tratamiento la frecuencia de trastorno del sueño fue de $83.3 \%$ y para los que llevan un año o menos con el tratamiento esta frecuencia fue de $50 \%(p<0.05)$ (Tabla 2).

\section{DISCUSIÓN}

En un estudio realizado en niños de siete a nueve años, que viven en un medio urbano, se analizó la presencia de asma y trastorno del sueño aplicando un cuestionario de hábitos del sueño para niños, el cual evalúa parámetros parecidos al utilizado en nuestro estudio, en su caso la presencia de trastornos del sueño fue de $86 \%$, el cual resultó mayor al referido en el presente estudio. ${ }^{15}$
Pablo E Brockmann y colaboradores analizaron la asociación entre asma y trastornos del sueño en menores de 18 años, encontraron que los trastornos del sueño fueron significativamente más frecuentes en niños con asma con una RM $=1.9$ [1.7:2.2]. ${ }^{16}$ De igual manera, otro estudio realizado por E Ridolo CC y colegas, en la que evaluaron la escala de trastornos de sueño de Bruni, encontraron que $62.3 \%$ de los niños estudiados tuvieron un puntaje $\geq 39,{ }^{12}$ mientras que en el estudio se encontró una frecuencia mayor de los pacientes con asma que presentan trastornos del sueño y un riesgo más elevado.

En otra revisión sistemática ${ }^{17}$ realizada para describir la relación entre asma y trastornos del sueño en menores de 18 años, en la cual los criterios de inclusión y exclusión son parecidos a los realizados, inclusive teniendo un grupo control, incluyeron estudios en los que aplicaron cuestionarios para evaluar trastornos del sueño; ninguno de los artículos que se encontraron fueron realizados en Latinoamérica; de los 32 estudios analizados encontraron una prevalencia de trastornos del sueño de $41.8 \%$, la cual es inferior a la observada en el presente estudio con una prevalencia en siete de cada 10 niños.

\section{BIBLIOGRAFÍA}

1. Organización Mundial de la Salud [Internet]. Asma, 2017 [Citado 18 Junio 2015]. Available from: www.who. int $>$ respiratory $>$ asthma.

2. Fermán IA, Nava FV, Guzmán FJ, Ochoa DL, Gómez MD. Alergias, asma, rinitis y eczema en niños y su relación con la lactancia, dieta y uso de medicamentos. Revista electrónica: Medicina, Salud y Sociedad. 2012; 2 (2): 23.

3. Mancilla-Hernández E, Medina-Ávalos MA, BarnicaAlvarado $\mathrm{RH}$, Soto-Candia $\mathrm{D}$ et al. Prevalencia de asma y determinación de los síntomas como indicadores de riesgo. Revista Alergia México. 2015; 62 (4): 271-278.

4. Ramagopal M, Scharf SM, Roberts DW, Blaisdell CJ. Obstructive sleep apnea and history of asthma in snoring children. Sleep Breath. 2008; 12 (4): 381-392. 
Vol. 29, Núm. 2・ Mayo-Agosto 2020

5. Labarca G, Cruz R, Descalzi F. Compromiso multisistémico en apnea obstructiva del sueño. Rev Méd Chile. 2014; 142 (6): 748-757.

6. Secretaría de Salud. Guía de práctica clínica diagnóstico y tratamiento de los trastornos del sueño. México, 2010 [Cited 2018].

7. Contreras SA. Sueño a lo largo de la vida y sus implicancias en salud. Revista Médica Clínica Las Condes. 2013; 24 (3): 341-349.

8. Alonso-Álvarez ML, Canet T, Cubell-Alarco M, Estivill E, Fernández-Julián E, Gonzal D et al. Documento de consenso del síndrome de apneas-hipoapneas durante el sueño en niños. Arch Bronconeumol. 2011; 47: 2-18.

9. Becerra MH. Epidemiología del asma. Neumología y Cirugía de Tórax. 2009; 68 (S2): S91-97.

10. Li L, Xu Z, Yan C, Jiang F, Tong S, Shen X, Li S. Sleepdisordered breathing and asthma: evidence from a large multicentric epidemiological study in China. Respir Res. 2015; 16 (1): 56.

11. Carotenuto M, Bruni O, Santoro N, del Giudice EM, Perrone $\mathrm{L}$, Pascotto A. Waist circumference predicts the occurrence of sleep-disordered breathing in obese children and adolescents: a questionnaire-based study. Sleep Med. 2006; 7 (4): 357-361.

12. Ridolo E, Caffarelli C, Olivieri E, Montagni M, Incorvaia C, Baiardini I et al. Quality of sleep in allergic children and their parents. Allergol Immunopathol. 2015; 43 (2): 180184.

13. Van Maanen A, Wijga AH, Gehring U, Postma DS, Smit HA, Oort FJ et al. Sleep in children with asthma: results of the PIAMA study. Eur Respir J. 2013; 41 (4): 832-837.
14. Lomeli HA, Pérez-Olmos I, Talero-Gutiérrez C, Moreno CB, González-Reyes R, Palacios L et al. Escalas y cuestionarios para evaluar el sueño: una revisión. Actas Esp Psiquiatr. 2008; 36 (1): 50-59.

15. Koinis-Mitchell D, Kopel SJ, Boergers J, Ruaid EL, Esteban $\mathrm{CA}$, Seifer $\mathrm{R}$ et al. Asthma, allergic rhinitis, and sleep problems in urban children. J Clin Sleep Med. 2015; 11 (2): 101.

16. Brockmann PE, Bertrand P, Castro-Rodriguez JA. Influence of asthma on sleep disordered breathing in children: a systematic review. Sleep Med Rev. 2014; 18 (5): 393-397.

17. Sánchez T, Castro-Rodríguez JA, Brockmann PE. Sleepdisordered breathing in children with asthma: a systematic review on the impact of treatment. J Asthma Allergy. 2016; 9: 83.

Financiamiento y conflicto de intereses: No existieron fuentes de financiamiento para la investigación y no existe conflicto de intereses por parte de los autores.

Dirección para correspondencia:

M.C. Luis Sandoval Jurado

Coordinación Auxiliar Médica

de Investigación en Salud

Av. Instituto Politécnico Nacional,

Esquina con Kinik, Región Núm. 509, 77500,

Cancún, Q. Roo, México.

Tel: (998) 2678701

E-mail: sanju.luis@gmail.com, luis.sandovalj@imss.gob.mx 